This item was submitted to Loughborough's Research Repository by the author.

Items in Figshare are protected by copyright, with all rights reserved, unless otherwise indicated.

\title{
Under-actuated back-stepping: An introduction
}

PLEASE CITE THE PUBLISHED VERSION

https://doi.org/10.1109/CDC.2018.8619350

PUBLISHER

(c) IEEE

VERSION

AM (Accepted Manuscript)

LICENCE

CC BY-NC-ND 4.0

REPOSITORY RECORD

Jiang, Jingjing, and Alessandro Astolfi. 2018. “Under-actuated Back-stepping: An Introduction”. figshare. https://hdl.handle.net/2134/35431. 


\title{
Under-Actuated Back-Stepping: An Introduction
}

\author{
Jingjing Jiang ${ }^{1}$ and Alessandro Astolfi ${ }^{2}$
}

\begin{abstract}
The stabilization problem for a class of underactuated systems is solved. This is achieved via a novel backstepping based method that we call under-actuated backstepping. The method is developed for linear under-actuated systems first and then extended to nonlinear systems via an example. Numerical simulations are given to demonstrate the effectiveness of the proposed under-actuated back-stepping method.
\end{abstract}

\section{INTRODUCTION}

Under-actuation is a technical term describing systems (usually mechanical systems) with fewer control inputs than degrees-of-freedom. In particular, the state of an underactuated mechanical system is unable to follow arbitrary reference trajectories. As a result, when compared to the control of fully-actuated (mechanical) system, the control design for under-actuated systems is more challenging. One of the broadly used methods to solve control problems for under-actuated systems is based on the linearization technique [1], [2]. Moreover, as energy is a fundamental concept in the control of mechanical systems, "energyshaping control" (also known as Passivity-Based Control (PBC)), first proposed by Takegaki et al. [3], is also a popular way to control mechanical systems [4], [5]. The main drawback of PBC is its difficult applicability, especially for under-actuated systems. The paper [6] has utilized adaptive control to deal with under-actuated systems, while the papers [7], [8] have applied Lyapunov-based control to mechanical systems. Finally, Sliding Mode Control (SMC) is another method used to control mechanical systems [9]. However, for many mechanical systems it is difficult to find a surface suitable for the application of SMC. In addition, other control techniques, such as optimal control [10] and hybrid and switching-based control [11], [12], have also been used. More recently, robust control has been exploited for the study of mechanical systems to deal with model uncertainties, nonholonomic constraints and disturbances [13], [14]. To sum up, apart from linearization based control techniques, all other methods have been mainly used to stabilize particular classes of under-actuated systems.

This work has been done when Jingjing works in KIOS project and has been supported by the European Unions Horizon 2020 research and innovation programme under grant agreement No 739551 (KIOS CoE).

${ }^{1} \mathrm{~J}$. Jiang is with the Department of Aeronautical and Automotive Engineering, Loughborough University, UK, E-mail: J. Jiang2@lboro.ac.uk

${ }^{2} \mathrm{~A}$. Astolfi is with the Dept. of Electrical and Electronic Engineering, Imperial College London, London, SW7 2AZ, UK and the DICII, University of Roma "Tor Vergata", Via del Politecnico 1, 00133 Rome, Italy, E-mail: a.astolfieimperial.ac.uk
The back-stepping method, a well-known control design technique developed in [15], [16], is a constructive method to design stabilizers for classes of nonlinear systems. The systems amenable to the application of back-stepping can be written in the so-called feedback form

$$
\begin{aligned}
& \dot{x}=f_{x}(x)+g_{x}(x) z, \\
& \dot{z}=f(x, z)+g(x) u,
\end{aligned}
$$

where $x(t) \in \mathbb{R}^{n}$ and $z(t) \in \mathbb{R}^{m}$ are the states and $u(t) \in \mathbb{R}^{m}$ is the input. In addition, $f_{x}(x): \mathbb{R}^{n} \longrightarrow$ $\mathbb{R}^{n}, g_{x}(x): \mathbb{R}^{n} \longrightarrow \mathbb{R}^{n \times m}, f(x, z): \mathbb{R}^{n+m} \longrightarrow \mathbb{R}^{m}$ and $g(x, z): \mathbb{R}^{n+m} \longrightarrow \mathbb{R}^{m \times m}$ are smooth mappings. Under the assumptions that $\operatorname{det}(g(x)) \neq 0$ for all $x \in \mathbb{R}^{n}$ and $u_{x}$ stabilizes the zero equilibrium of the subsystem

$$
\dot{x}=f_{x}(x)+g_{x}(x) u_{x}(x),
$$

we can always find a state feedback controller $u=u(x, z)$, through back-stepping, such that the origin of the closedloop system is globally asymptotically stable [15], [16], [17], [18]. Note that in this "fully-actuated" case, that is the case in which the dimension of $z$ and of $u$ are the same, one can select any stabilizer $u_{x}$ in the initial step of back-stepping.

The main contribution of the paper is as follows. The paper proposes a novel method, the under-actuated back-stepping method, which is inspired by standard back-stepping, to solve the stabilization problem for classes of under-actuated (mechanical) system. The proposed method is explained in detail for linear time-invariant under-actuated systems first and then the nonlinear extension is discussed via an example.

The rest of the paper is organized as follows. In Section II the dynamics of two classes of linear under-actuated system are given and the stabilization problem for these systems is formulated. Solutions to the stated problems are presented in Section III, in which the stability properties of the resulting closed-loop systems with the controllers developed by the proposed methodology are given. In addition, one numerical example to illustrate how under-actuated backstepping works is provided. Section IV studies how the design procedure developed for linear systems can be extended to nonlinear systems and uses the Inertia Wheel Pendulum system to demonstrate the effectiveness of the method. Finally, conclusions and suggestions for future work are given in Section V.

\section{PROBLEM STATEMENT}

This section formulates the stabilization problem for two classes of under-actuated linear systems. The under-actuated 
back-stepping based solutions for these stabilization problems is given in Section III.

The term under-actuated is used informally to denote systems in cascaded form for which the number of input is strictly less than half the number of states. These systems arise when linearizing under-actuated mechanical systems.

Problem 1: Consider an under-actuated system, the dynamics of which are described by the equations

$$
\begin{aligned}
& \dot{x}=z, \\
& \dot{z}=A x+B u,
\end{aligned}
$$

with $A \in \mathbb{R}^{n \times n}, B \in \mathbb{R}^{n \times m}$, states $x(t) \in \mathbb{R}^{n}$ and $z(t) \in$ $\mathbb{R}^{n}$ and input $u(t) \in \mathbb{R}^{m}$, with $0<m<n$. Assume that $\operatorname{rank}(B)=m$ hence, without loss of generality, let $B=$ $\left[I_{m}, 0_{m \times(n-m)}\right]^{T}$. Find a controller

$$
u=H_{1} x+H_{2}(z-K x),
$$

with $H_{1} \in \mathbb{R}^{m \times n}, H_{2} \in \mathbb{R}^{m \times n}$ and $K \in \mathbb{R}^{n \times n}$, such that the closed-loop system (2)-(3) is asymptotically stable.

Problem 2: Consider an under-actuated system, the dynamics of which are described by the equations

$$
\begin{aligned}
& \dot{x}=F x+G z, \\
& \dot{z}=A x+E z+B u,
\end{aligned}
$$

with $F \in \mathbb{R}^{n \times n}, G \in \mathbb{R}^{n \times n}, A \in \mathbb{R}^{n \times n}, E \in \mathbb{R}^{n \times n}, B \in$ $\mathbb{R}^{n \times m}$, states $x(t) \in \mathbb{R}^{n}$ and $z(t) \in \mathbb{R}^{n}$ and control input $u(t) \in \mathbb{R}^{m}$, with $0<m<n$. Assume that $\operatorname{rank}(B)=m$ and $\operatorname{det}(G) \neq 0$. Hence, without loss of generality, assume that $G B=\left[I_{m}, 0_{(n-m) \times m}^{T}\right]^{T}$. Find a controller

$$
u=H_{1} x+H_{2}(G z-K x),
$$

with $H_{1} \in \mathbb{R}^{m \times n}, H_{2} \in \mathbb{R}^{m \times n}$ and $K \in \mathbb{R}^{n \times n}$, such that the closed-loop system (4)-(5) is asymptotically stable.

It is tacitly assumed that the system (2) and (4) are controllable, hence these can be stabilized using a linear state feedback. The goal of the paper is therefore not to merely stabilize these systems, but to propose a modular design inspired by back-stepping and hence applicable, in principle, to nonlinear systems (see the example in Section IV).

\section{MAIN RESULTS}

In this section we give solutions to the Problems 1 and 2 stated in Section II. The corresponding under-actuated backstepping algorithms used to find the controllers are given in Algorithms 1 and 2, respectively. Finally, an example is given at the end of this section to show how the algorithms work.

We begin with Problem 1, as the dynamics of system (2) can be regarded as a special case of the dynamics of the systems studied in Problem 2. Algorithm 1 can be used to find the controller (3) and the existence of the matrix $K$ is proved in Lemma 1.

$\frac{\text { Algorithm 1: Solution To Problem 1 }}{\text { procedure UNDER-ACTUATED BACK-STEPPING }}$

Step 1: Find a matrix $K \in \mathbb{R}^{n \times n}$ such that the conditions

$$
\lambda(K) \in \mathbb{C}^{-},
$$

$(K, B)$ controllable,

$$
J_{1}\left(A-K^{2}\right)=0_{n \times n}
$$

where

$$
J_{1}=\left[\begin{array}{cc}
0_{m \times m} & 0_{m \times(n-m)} \\
0_{(n-m) \times m} & I_{(n-m) \times(n-m)}
\end{array}\right]
$$

hold.

Step 2: Set

$$
H_{1}=-J_{2}\left(A-K^{2}\right)
$$

with

$$
J_{2}=\left[\begin{array}{ll}
I_{m \times m}, & 0_{(n-m) \times m}
\end{array} .\right.
$$

Step 3: Select $H_{2}$ such that

$$
\lambda\left(-K+B H_{2}\right) \in \mathbb{C}^{-} .
$$

end procedure

Lemma 1: Consider the system (2) with $m=1$, yielding $B=[1,0,0, \ldots, 0]^{T}$. Then there exists a matrix $K$ such that conditions (6) to (8) in Algorithm 1 hold.

Proof: The matrices $A$ and $K$ can be written as

$$
A=\left[\begin{array}{ll}
a_{11} & A_{12} \\
A_{21} & A_{22}
\end{array}\right], \quad K=\left[\begin{array}{cc}
k_{11} & K_{12} \\
K_{21} & K_{22}
\end{array}\right],
$$

where $a_{11} \in \mathbb{R}, A_{12}^{T} \in \mathbb{R}^{n-1}, A_{21} \in \mathbb{R}^{n-1}, A_{22} \in$ $\mathbb{R}^{(n-1) \times(n-1)}, \quad k_{11} \in \mathbb{R}, K_{12}^{T} \in \mathbb{R}^{n-1}, K_{21} \in \mathbb{R}^{n-1}$ and $K_{22} \in \mathbb{R}^{(n-1) \times(n-1)}$. Since $(A, B)$ is controllable, then $\left(A_{22}, A_{21}\right)$ is controllable. Hence, there is at least one element of $A_{21}$ which is nonzero. Without loss of generality, we assume that the first element of $A_{21}$, denoted as $a_{2,1}$, is nonzero.

Note that (7) is equivalent to controllability of $\left(K_{22}, K_{21}\right)$. Pick $K_{22}$ and $K_{21}$ to satisfy the above contrallability condition. One such a choice is $K_{21}=[\alpha, 0,0, \ldots, 0]^{T}$, with nonzero $\alpha$, and

$$
K_{22}=\left[\begin{array}{cccc}
\beta_{1,1} & \beta_{1,2} & \cdots & \beta_{1, n-1} \\
\beta_{2,1} & \beta_{2,2} & \cdots & \beta_{2, n-1} \\
\vdots & \vdots & \vdots & \vdots \\
\beta_{n-1,1} & \beta_{n-1,2} & \cdots & \beta_{n-1, n-1}
\end{array}\right]
$$

with $\beta_{i, j}=0$, for all $i \neq j$ and $i \neq(j+1)$, and $\beta_{i, j} \neq 0$, for all $i=j$ and $i=(j+1)$. Exploiting (8) $k_{11}$ and $K_{12}$ can then be calculated as

$$
\begin{aligned}
k_{11} & =\left(K_{21}^{T} K_{21}\right)^{-1} K_{21}^{T}\left[A_{21}-K_{22} K_{21}\right], \\
K_{12} & =\left(K_{21}^{T} K_{21}\right)^{-1} K_{21}^{T}\left[A_{22}-K_{22}^{2}\right] .
\end{aligned}
$$


Note that if $\beta_{2,1}=0$ then the eigenvalues of the matrix $K$, denoted as $\lambda_{1}, \lambda_{2}, \ldots, \lambda_{n}$, are such that $\lambda_{3}=\beta_{2,2}, \lambda_{4}=$ $\beta_{3,3}, \ldots, \lambda_{n}=\beta_{n-1, n-1}$, and

$$
\lambda_{1}+\lambda_{2}=\frac{a_{2,1}}{\alpha}, \quad \lambda_{1} \lambda_{2}=\frac{a_{2,1} \beta_{1,1}}{\alpha}-a_{2,2},
$$

where $a_{2,2}$ denotes the element on the second row and second column of the matrix $A$.

Selecting

$$
\begin{array}{r}
\beta_{1,1}=-a_{2,2}-1, \beta_{i, i}<0, \forall i \in\{2,3, \ldots, n-1\}, \\
\alpha=-a_{2,1}, \beta_{i+1, i} \neq 0, \forall i \in\{2,3, \ldots, n-2\},
\end{array}
$$

and noting that the eigenvalues are robust, i.e. their position is only modified by a small change in parameters, one could select $\beta_{2,1} \neq 0$ and small to satisfy (6) and (7), hence the claim.

Remark 1: Lemma 1 shows that $K$ in Algorithm 1 exists in the case $m=1$. It is trivial to extend the result to the case $m \geq 2$.

The stability properties for the closed-loop system (2)-(3) can then be summarized as follows.

Proposition 1: Consider the under-actuated system (2) controlled with the feedback law given by (3) with $K \in$ $\mathbb{R}^{n \times n}, H_{1} \in \mathbb{R}^{m \times n}$ and $H_{2} \in \mathbb{R}^{m \times n}$ as in Algorithm 1 . Then the closed-loop system (2)-(3) is asymptotically stable.

Proof: To begin with define the new variable $\Delta z$ as $\Delta z=z-K x$. Using the new variable $\Delta z$ the system (2) can be rewritten as

$$
\begin{aligned}
\dot{x} & =K x+\Delta z, \\
\dot{\Delta} z & =\left(A-K^{2}\right) x-K \Delta z+B u .
\end{aligned}
$$

Substituting the control law (3) into equations (13) yields

$$
\begin{aligned}
\dot{x} & =K x+\Delta z, \\
\dot{\Delta} z & =\left(A-K^{2}+B H_{1}\right) x+\left(B H_{2}-K\right) \Delta z .
\end{aligned}
$$

By equations (8) and (10) one has

$$
\begin{aligned}
\dot{x} & =K x+\Delta z, \\
\dot{\Delta} z & =\left(B H_{2}-K\right) \Delta z .
\end{aligned}
$$

The condition (7) implies that there exists $H_{2}$ such that (11) holds. Hence, there exists a symmetric positive definite matrix $Q$ such that

$$
\left(B H_{2}-K\right)^{T} Q+Q\left(B H_{2}-K\right)=-I .
$$

Similarly, by condition (6), there exists a symmetric positive definite matrix $P$ such that

$$
K^{T} P+P K=-I .
$$

Let $\epsilon$ be a sufficiently small positive constant. Consider the Lyapunov function candidate

$$
L(x, z)=\epsilon x^{T} P x+\Delta z^{T} Q \Delta z .
$$

Its time derivative along the trajectories of the closed-loop system is such that

$$
\begin{aligned}
\dot{L}= & \epsilon\left(x^{T} K^{T}+\Delta z^{T}\right) P x+\epsilon x^{T} P(K x+\Delta z) \\
& +\Delta z^{T}\left[\left(B H_{2}-K\right)^{T} Q+Q\left(B H_{2}-K\right)\right] \Delta z \\
\leq & \epsilon x^{T}\left(K^{T} P+P K\right) x+\frac{\epsilon}{2} x^{T} x+2 \epsilon \Delta z^{T} P^{T} P \Delta z \\
& +\Delta z^{T}\left[\left(B H_{2}-K\right)^{T} Q+Q\left(B H_{2}-K\right)\right] \Delta z \\
\leq & -\frac{\epsilon}{2} x^{T} x-\left(1-2 \epsilon \bar{\sigma}_{P}^{2}\right) \Delta z^{T} \Delta z,
\end{aligned}
$$

where $\bar{\sigma}_{P}$ denotes the maximum singular value of the matrix $P$. Therefore, $\dot{L} \leq 0$ for all $0<\epsilon<\frac{1}{2 \bar{\sigma}_{P}^{2}}$. In addition,

$$
\dot{L}=0 \Longleftrightarrow x=z=0,
$$

hence the claim.

Remark 2: If the system (2) is fully-actuated, i.e. $m=n$, then trivially conditions (7) and (8) hold. Therefore, in Step 1 the matrix $K$ only needs to satisfy condition (6), consistently with standard back-stepping, i.e. any "stabilizing" feedback can be selected in the initial step of back-stepping.

Problem 2 is a generalization of Problem 1 and can also be solved using the under-actuated back-stepping method. The steps of the control design are detailed in Algorithm 2. The existence of the matrix $K$ and the formal properties of the closed-loop system (4)-(5) are given in Lemma 2 and Proposition 2, respectively.

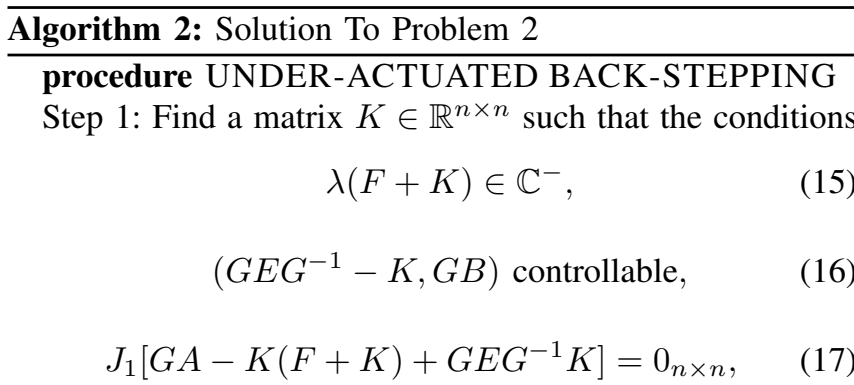

where

$$
J_{1}=\left[\begin{array}{cc}
0_{m \times m} & 0_{m \times(n-m)} \\
0_{(n-m) \times m} & I_{(n-m) \times(n-m)}
\end{array}\right],
$$

hold.

Step 2: Set

$$
H_{1}=-J_{2}\left[G A-K(F+K)+G E G^{-1} K\right],
$$

with

$$
J_{2}=\left[\begin{array}{ll}
I_{m \times m}, & 0_{(n-m) \times m}
\end{array} .\right.
$$

Step 3: Select $H_{2}$ such that

$$
\lambda\left(G E G^{-1}-K+G B H_{2}\right) \in \mathbb{C}^{-} .
$$

\section{end procedure}

Lemma 2: Consider the system (4) with $m=1$, yielding $B=[1,0,0, \ldots, 0]^{T}$. Then there exists a matrix $K$ such that conditions (15) to (17) hold. 
Proof: Similarly to the proof of Lemma 1, we rewrite the matrices $A$ and $K$ as in (12) and the matrices $E$ and $F$ as

$$
E=\left[\begin{array}{cc}
e_{11} & E_{12} \\
E_{21} & E_{22}
\end{array}\right], \quad F=\left[\begin{array}{cc}
f_{11} & F_{12} \\
F_{21} & F_{22}
\end{array}\right]
$$

where $e_{11} \in \mathbb{R}, E_{12}^{T} \in \mathbb{R}^{n-1}, E_{21} \in \mathbb{R}^{n-1}, E_{22} \in$ $\mathbb{R}^{(n-1) \times(n-1)}, f_{11} \in \mathbb{R}, F_{12}^{T} \in \mathbb{R}^{n-1}, F_{21} \in \mathbb{R}^{n-1}, F_{22} \in$ $\mathbb{R}^{(n-1) \times(n-1)}$. Since $G$ is invertible, without loss of generality we can assume $G=I_{n}$. System (4) is controllable, indicating that

$$
\left(\left[\begin{array}{ccc}
F_{11} & F_{12} & 0_{n-1}^{T} \\
F_{21} & F_{22} & I_{n-1} \\
A_{21} & A_{22} & E_{22}
\end{array}\right],\left[\begin{array}{c}
1 \\
0_{n-1} \\
E_{21}
\end{array}\right]\right) \text { is controllable, }
$$

hence $\left(E_{21}+F_{21}\right) \neq 0_{n-1}$. Without loss of generality, we can assume that its first element is nonzero. Choose $K_{21}$ and $K_{22}$ such that $E_{21}-K_{21}=[\alpha, 0,0, \ldots, 0]^{T}$, with nonzero $\alpha$, and

$$
E_{22}-K_{22}=\left[\begin{array}{cccc}
\beta_{1,1} & \beta_{1,2} & \cdots & \beta_{1, n-1} \\
\beta_{2,1} & \beta 2,2 & \cdots & \beta_{2, n-1} \\
\vdots & \vdots & \vdots & \vdots \\
\beta_{n-1,1} & \beta_{n-1,2} & \cdots & \beta_{n-1, n-1}
\end{array}\right],
$$

with $\beta_{i, j}=0$, for all $i \neq j$ and $i \neq(j+1)$. Exploiting (17) $k_{11}$ and $K_{12}$ can then be calculated as

$$
\begin{aligned}
k_{11}= & {\left[\left(K_{21}-E_{21}\right)^{T}\left(K_{21}-E_{21}\right)\right]^{-1}\left(K_{21}-E_{21}\right)^{T} } \\
& \times\left[A_{21}-K_{21} f_{11}-K_{22} F_{21}-K_{22} K_{21}+E_{22} K_{21}\right], \\
K_{12}= & {\left[\left(K_{21}-E_{21}\right)^{T}\left(K_{21}-E_{21}\right)\right]^{-1}\left(K_{21}-E_{21}\right)^{T} } \\
& \times\left[A_{22}-K_{21} F_{12}-K_{22} F_{22}-K_{22}^{2}+E_{22} K_{22}\right] .
\end{aligned}
$$

Let the eigenvalues of $(F+K)$ be $\lambda_{1}, \lambda_{2}, \ldots, \lambda_{n}$. Then by choosing $\beta_{i, i} \gg 0$, for all $i \in\{2,3, \ldots, n-1\}$ we have

$$
\begin{aligned}
\lambda_{1}+\lambda_{2} \approx & \frac{e_{2,1}+f_{2,1} \beta_{1,1}+e_{2,2}+f_{2,2}}{\alpha}, \\
& +\frac{a_{2,1}-\Sigma_{i=1}^{n}\left(e_{2, i} f_{i, 1}\right)}{\alpha}, \\
\lambda_{1} \lambda_{2} \approx & -\beta_{1,1}^{2}+\left(e_{2,2}+f_{2,2}-\frac{e_{2,1}+f_{2,1}}{\alpha} e_{2,2}\right) \beta_{1,1} \\
& -\frac{a_{2,1}-\Sigma_{i=1}^{n}\left(e_{2, i} f_{i, 1}\right)}{\alpha} \beta_{1,1}+\frac{e_{2,1}+f_{2,1}}{\alpha} f_{1,2} e_{2,1} \\
& +\frac{\left[a_{2,1}-\Sigma_{i=1}^{n}\left(e_{2, i} f_{i, 1}\right)\right]\left(e_{2,2}+f_{2,2}\right)}{\alpha} \\
& +\frac{e_{2,1}+f_{2,1}}{\alpha}\left(\Sigma_{i=4}^{n}\left(e_{2, i} e_{i, 2}\right)-a_{3,2}-e_{2,2}^{2}\right) \\
& +\frac{e_{2,1}+f_{2,1}}{\alpha} e_{2,3}\left(e_{3,2}-\beta_{2,1}\right),
\end{aligned}
$$

where $e_{i, j}$ and $f_{i, j}$ denote the element of the $j^{\text {th }}$-column $i^{t h}$ row of the matrix $E$ and $F$, respectively, and $\lambda_{i} \approx-\beta_{i-1, i-1}$ for all $i \in\{3,4, \ldots, n\}$. Since $e_{2,1}+f_{2,1} \neq 0$, it is always possible to find $\alpha$ and $\beta_{1,1}$ such that $\lambda_{1}<0$ and $\lambda_{2}<0$, hence the claim.

Proposition 2: Consider the under-actuated system (4) with the controller (5) with $K \in \mathbb{R}^{n \times n}, H_{1} \in \mathbb{R}^{m \times n}$ and $H_{2} \in$
$\mathbb{R}^{m \times n}$ as in Algorithm 2. Then the zero equilibrium of the closed-loop system is asymptotically stable.

Proof: To begin with define the new variable

$$
\Delta z=G z-K x
$$

Recall that $G^{-1}$ exists since $\operatorname{det}(G) \neq 0$. Using the new variable $\Delta z$ the system (4) can be rewritten as

$$
\begin{aligned}
\dot{x}= & (F+K) x+\Delta z, \\
\dot{\Delta} z= & {\left[G A-K(F+K)+G E G^{-1} K\right] x } \\
& +\left(G E G^{-1}-K\right) \Delta z+G B u .
\end{aligned}
$$

Substituting the control law (5), together with equations (18) and (19) into the equation (22), yields

$$
\begin{aligned}
\dot{x} & =(F+K) x+\Delta z, \\
\dot{\Delta} z & =\left(G E G^{-1}-K+G B H_{2}\right) \Delta z .
\end{aligned}
$$

The condition (16) implies that we can always find a matrix $\mathrm{H}_{2}$ such that (19) holds. Similarly to the proof of Proposition 1 , there exists matrices $P$ and $Q$ such that

$$
\begin{array}{r}
P=P^{T}>0, \quad Q=Q^{T}>0, \\
M^{T} Q+Q M=-I_{n}, \\
(F+K)^{T} P+P(F+K)=-I_{n},
\end{array}
$$

where $M=G E G^{-1}-K+G B H_{2}$.

Again, let $\epsilon$ be a sufficiently small positive constant. Consider the Lyapunov function candidate (14), the time derivative of which along the trajectories of the closed-loop system is such that

$$
\begin{aligned}
\dot{L}= & \epsilon\left[x^{T}(F+K)^{T}+\Delta z^{T}\right] P x+\epsilon x^{T} P[(F+K) x+\Delta z] \\
& +\Delta z^{T}\left(G E G^{-1}-K+G B H_{2}\right)^{T} Q \Delta z \\
& +\Delta z^{T} Q\left(G E G^{-1}-K+G B H_{2}\right) \Delta z \\
\leq & \epsilon x^{T}\left[(F+K)^{T} P+P(F+K)\right] x+\frac{\epsilon}{2} x^{T} x \\
& +2 \epsilon \Delta z^{T} P^{T} P \Delta z \\
& +\Delta z^{T}\left(G E G^{-1}-K+G B H_{2}\right)^{T} Q \Delta z \\
& +\Delta z^{T} Q\left(G E G^{-1}-K+G B H_{2}\right) \Delta z \\
\leq & -\frac{\epsilon}{2} x^{T} x-\left(1-2 \epsilon \bar{\sigma}_{P}^{2}\right) \Delta z^{T} \Delta z \leq 0,
\end{aligned}
$$

where $\bar{\sigma}_{P}$ is the maximum singular value of the matrix $P$, hence the claim.

Example 1: Consider the two degrees-of-freedom, underactuated linear system described by the equations

$$
\begin{aligned}
& \dot{x}=\left[\begin{array}{ll}
0 & 0 \\
0 & 1
\end{array}\right] x+\left[\begin{array}{ll}
1 & 0 \\
0 & 1
\end{array}\right] z, \\
& \dot{z}=\left[\begin{array}{ll}
0 & 0 \\
1 & 0
\end{array}\right] x+\left[\begin{array}{ll}
0 & 1 \\
0 & 0
\end{array}\right] z+\left[\begin{array}{l}
1 \\
0
\end{array}\right] u,
\end{aligned}
$$

with states $x(t) \in \mathbb{R}^{2}$ and $z(t) \in \mathbb{R}^{2}$ and input $u(t) \in \mathbb{R}$. 
According to Algorithm 2 the first step is to find a matrix $K$ satisfying conditions (15), (16) and (17). One such a choice is given by

$$
K=\left[\begin{array}{rr}
0 & 4 \\
-\frac{1}{2} & -2
\end{array}\right]
$$

yielding

$$
H_{1}=[-1.5,-2]
$$

Then $H_{2}$ has to be chosen such that (19) holds. One such a choice is given by

$$
H_{2}=[-3,-10],
$$

yielding the overall controller

$$
\begin{aligned}
u & =H_{1} x+H_{2}\left(\left[\begin{array}{ll}
1 & 0 \\
0 & 1
\end{array}\right] z-K x\right) \\
& =[-6.5,-10] x+[-3,-10] z .
\end{aligned}
$$

\section{TOWARds NONLINEAR UNDER-ACTUATED BACK-STEPPING}

In this section we discuss how the under-actuated backstepping method can be used to design stabilizers for nonlinear under-actuated systems. The dynamics of a class of under-actuated mechanical systems can be described by the equations

$$
\begin{aligned}
& \dot{x}=z, \\
& \dot{z}=A(x) x+E z+B u,
\end{aligned}
$$

where the states $x(t) \in \mathbb{R}^{n}$ and $z(t) \in \mathbb{R}^{n}$ denotes the position and velocity vectors, respectively, while the input $u(t) \in \mathbb{R}^{m}$ represents the force or torque vector. The mapping $A(x): \mathbb{R}^{n} \longrightarrow \mathbb{R}^{n \times n}$ is smooth and $B \in \mathbb{R}^{n \times m}$ and $E \in \mathbb{R}^{n \times n}$ are constant matrices. Assume that $\operatorname{rank}(B)=m$ hence, without loss of generality, let $B=\left[I_{m}, 0_{(n-m) \times m}^{T}\right]^{T}$. To find a control law $u(x, z)$ such that the zero equilibrium of the closed-loop system is asymptotically stable we can exploit the under-actuated back-stepping method developed.

The first step is to find the mappings $K: \mathbb{R}^{n} \longrightarrow \mathbb{R}^{n}$ and $H_{2}: \mathbb{R}^{m} \longrightarrow \mathbb{R}^{n}$ such that the following properties hold.

P1) $\frac{\partial\left(B H_{2}(x)-K(x)\right)}{\partial x}=0_{n \times n}$.

P2) The point $x=0$ and $\Delta z=0$ are globally asymptotically stable equilibrium of the subsystems

$$
\dot{x}=K(x)
$$

and

$$
\dot{\Delta} z=\left[E-\frac{\partial K(x)}{\partial x}+B H_{2}(x)\right] \Delta z,
$$

uniformly in $x$, respectively.

P3) $J_{1}\left(A(x)+E K(x)-\frac{\partial K(x)}{\partial x} K(x)\right)=0_{n \times n}$, where $J_{1}$ is defined as in (9).
The second step of the method requires, in this case, to calculate $H_{1}: \mathbb{R}^{m} \longrightarrow \mathbb{R}^{n}$ as

$$
H_{1}(x)=-J_{2}\left(A(x)+E K(x)-\frac{\partial K(x)}{\partial x} K(x)\right),
$$

where $J_{2}$ is as given in (18).

Finally, globally asymptotic stability of the zero equilibrium of the system (25) in closed-loop with the control law

$$
u=H_{2}(x)(z-K(x))+H_{1}(x)
$$

can be proved by using a Lyapunov function candidate of the form

$$
L(x, z)=(z-K(x) x)^{T} Q(x)(z-K(x) x)+\epsilon x^{T} P(x) x,
$$

with $\epsilon>0$ and sufficiently small and $P: \mathbb{R}^{n} \longrightarrow \mathbb{R}^{n \times n}$ and $Q: \mathbb{R}^{n} \longrightarrow \mathbb{R}^{n \times n}$ positive definite.

The following example is used to illustrate how underactuated back-stepping can be applied to nonlinear systems.

Example 2: Consider an inertia wheel pendulum, the dynamics of which are described by the equations [1]

$$
\begin{aligned}
& \dot{x}=z, \\
& \dot{z}=\left[\begin{array}{c}
u, \\
\left(b_{2}-b_{1}\right) M \sin x_{1}
\end{array}\right],
\end{aligned}
$$

where

$$
\begin{aligned}
& M=\frac{\left(m_{1} l_{1}+m_{2} l_{2}\right) g}{I_{1}+m_{1} l_{1}^{2}+m_{2} l_{2}^{2}}, \quad b_{1}=\frac{1}{I_{1}+m_{1} l_{1}^{2}+m_{2} l_{2}^{2}}, \\
& b_{2}=\frac{1}{I_{2}}+\frac{1}{I_{1}+m_{1} l_{1}^{2}+m_{2} l_{2}^{2}}, \quad u=M \sin x_{1}-b_{1} \tau
\end{aligned}
$$

$x=\left[q_{1}, b_{2} q_{1}+b_{1} q_{2}\right]^{T}$ and $z$ are states of the system, while $u$ is the control input. $q_{1}$ and $q_{2}$ represent the pendulum angle and the wheel angle, respectively. Note that $I_{1}$ and $I_{2}$ denote the moment of inertia of the pendulum around its center of mass and the moment of inertia of the wheel (plus actuator's rotor), respectively; $m_{1}$ and $m_{2}$ represent the mass of the rod and the mass of the inertia wheel plus the mass of the actuator, respectively; $l_{1}$ and $l_{2}$ describes the distance to the center of mass of the rod and the length of the pendulum, respectively. In addition, $\tau$ denotes the torque generated by the actuator acting between the wheel and the pendulum, and $g$ is the gravity constant. Suppose that the parameter values are given as: $b_{1}=1.5 /\left(\mathrm{kg} \times \mathrm{m}^{2}\right), b_{2}=$ $668.17 /\left(k g \times m^{2}\right), M=74.0 / s^{2}$.

Based on the analysis given in Section IV, the first step is to find $K(x)=\left[k_{1}(x), k_{2}(x)\right]^{T}$, where $k_{1}(x): \mathbb{R}^{2} \rightarrow \mathbb{R}$ and $k_{2}(x): \mathbb{R}^{2} \rightarrow \mathbb{R}$, and $H_{2}(x)$ such that P1) to P3) hold. One such a choice for $K(x)$ and $\tilde{u}(x, z) \triangleq H(x)(z-K(x))$ is given by

$$
\begin{aligned}
k_{2}(x)= & \frac{1}{2} \ln \frac{1-\frac{4}{\pi} x_{1}-\tanh \left(x_{2}\right)}{1+\frac{4}{\pi} x_{1}-\tanh \left(x_{2}\right)} \\
k_{1}(x)= & -\frac{\pi}{2\left(e^{x_{2}}+e^{-x_{2}}\right)^{2}} \ln \frac{1-\frac{4}{\pi} x_{1}-\tanh \left(x_{2}\right)}{1+\frac{4}{\pi} x_{1}-\tanh \left(x_{2}\right)} \\
& -\frac{\pi}{4}\left[1-\left(\frac{4}{\pi} x_{1}+\tanh \left(x_{2}\right)\right)^{2}\right] M \sin x_{1}
\end{aligned}
$$



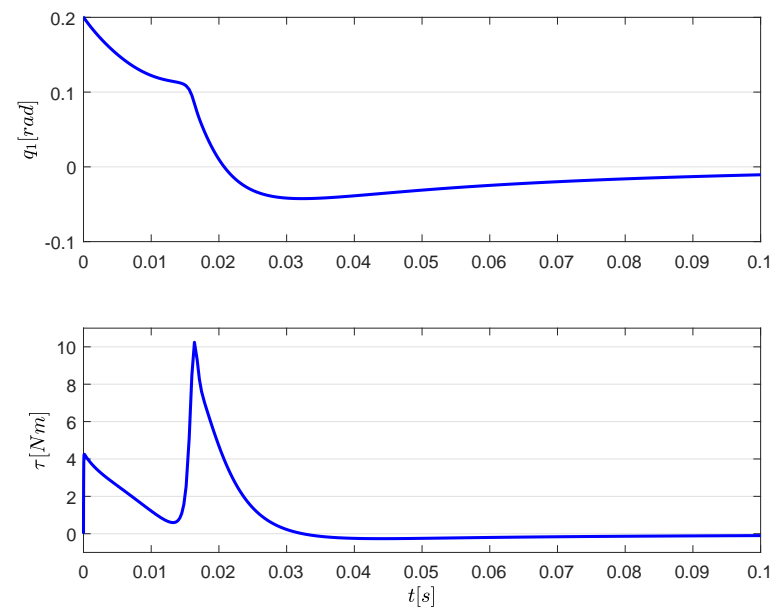

Fig. 1: Time histories of the pendulum angle $q_{1}$ and the torque generated by the actuator $\tau$ for the inertia wheel pendulum with the controller (31).

and

$$
\begin{aligned}
\tilde{u}(x, z)= & \frac{\partial k_{2}}{\partial x_{1}} \Delta_{2}+\dot{\Delta}_{1}^{*}-\gamma_{4}\left(\Delta_{1}-\Delta_{1}^{*}\right)+\frac{\partial k_{1}}{x_{1}} \Delta_{1} \\
& +\frac{\partial k_{1}}{x_{2}} \Delta_{2}
\end{aligned}
$$

where $\Delta_{1}=z_{1}-k_{1}(x), \Delta_{2}=z_{2}-k_{2}(x), \gamma_{4}>0$ and

$$
\Delta_{1}^{*}=-\frac{\frac{\partial k_{2}}{\partial x_{2}}}{\frac{\partial k_{2}}{\partial x_{1}}} \Delta_{2}-\gamma_{3} \Delta_{2},
$$

with $\gamma_{3}>0$, respectively. Note that $\frac{\partial k_{2}}{\partial x_{1}}<0$, hence $\Delta_{1}^{*}$ is well-defined.

The second step is to calculate $H_{1}(x)$ as

$$
H_{1}(x)=\frac{\partial k_{1}}{\partial x_{1}} k_{1}(x)+\frac{\partial k_{1}}{\partial x_{2}} k_{2}(x),
$$

with $k_{1}$ as given in (27).

Finally, the control input $u$ to the system (26) is calculated as

$$
u=H_{1}(x)+\tilde{u}(x, z),
$$

where $H_{1}(x)$ and $\tilde{u}(x, z)$ are calculated in (30) and (28), respectively.

Simulation results are given in Fig. 1 which shows that the controller (31) based on the proposed under-actuated back-stepping method is effective in stabilizing the zero equilibrium of the inertia wheel pendulum. Furthermore, the speed of convergence can be changed by choosing different values of $\gamma_{3}$ and $\gamma_{4}$.

As illustrated by the examples, the key step of the underactuated back-stepping method is the first step of the Algorithms 1 and 2. This restricts the selection of the initial stabilizing controller: such a restriction is not present in standard back-stepping.

\section{CONCLUSIONS}

This paper proposes a novel control method, the underactuated back-stepping method, to solve the stabilization problem for classes of under-actuated systems. The technique is studied in details for linear systems and its extension to nonlinear under-actuated systems is briefly discussed. Two numerical examples, one linear example given in Section III and the other nonlinear one given in Section IV, show the effectiveness of the proposed method and its main features. In future we will focus on the application of the under-actuated back-stepping method on classes to nonlinear systems.

\section{REFERENCES}

[1] S. Enev, "Feedback linearization control of the inertia wheel pendulum," Cybernetics and Information Technologies, vol. 14, no. 3, pp. 96-109, 2014.

[2] N. McClamroch and D. Wang, "Linear feedback control of position and contact force for a nonlinear constrained mechanism," ASME J. of Dynamic Systems, Measurement, and Control, vol. 112, no. 4, pp. 640-645, 1990.

[3] M. Takegaki and S. Arimoto, "A new feedback method for dynamic control of manipulators," ASME J. of Dynamic Systems, Measurement, and Control, vol. 103, no. 2, pp. 119-125, 1981.

[4] J. Romero, R. Ortega, and A. Donaire, "Energy Shaping of Mechanical Systems via PID Control and Extension to Constant Speed Tracking," IEEE Trans. on Automatic Control, vol. 61, no. 11, pp. 3551-3556, 2016.

[5] J. Acosta, R. Ortega, A. Astolfi, and A. Mahindrakar, "Interconnection and damping assignment passivity-based control of mechanical systems with underactuation degree one," IEEE Trans. on Automatic Control, vol. 50, no. 12, pp. 1936-1955, 2005.

[6] D. Pucci, F. Romano, and N. F., "Collocated adaptive control of underactuated mechanical systems," IEEE Trans. on Robotics, vol. 31, no. 6, pp. 1527-1536, 2015.

[7] M. Queiroz, D. Dawson, S. Nagarkatti, and F. Zhang, LyapunovBased Control of Mechanical Systems. New York, USA: Springer Science+Business Media, 2000.

[8] D. Wang and N. McClamroch, "Position and Force Control for Constrained Manipulator Motion: Lyapunov's Direct Method," IEEE Trans. on Robotics and Automation, vol. 9, no. 3, pp. 308-313, 1993.

[9] V. Utkin, J. Guldner, and J. Shi, Sliding Mode Control in ElectroMechanical Systems. CRC Press, 2009.

[10] I. Hussein and A. Bloch, "Optimal control of underactuated nonholonomic mechanical systems," IEEE Trans. on Automatic Control, vol. 53, no. 3, pp. 668-682, 2008.

[11] M. Zhang and T. Tarn, "A hybrid switching control strategy for nonlinear and underactuated mechanical systems," IEEE Trans. on Automatic Control, vol. 48, no. 10, pp. 1777-1782, 2003.

[12] R. Fierro, F. L. Lewis, and A. Lowe, "Hybrid control for a class of underactuated mechanical systems," IEEE Trans. on Systems, Man, and Cybernetics - Part A: Systems and Humans, vol. 29, no. 6, pp. 649-654, 1999.

[13] V. Sankaranarayanan and A. Mahindrakar, "Control of a class of underactuated mechanical systems using sliding modes," IEEE Trans. on Robotics, vol. 25, no. 2, pp. 459-467, 2009.

[14] C. Su and Y. Stepanenko, "Robust motion/force control of mechanical systems with classical nonholonomic constraints," IEEE Trans. on Automatic Control, vol. 39, no. 3, pp. 609-614, 1994.

[15] P. Kokotovic, "The joy of feedback: Nonlinear and adaptive," IEEE Control Systems Magazine, vol. 12, no. 3, pp. 7-17, 1992.

[16] M. Krstic, I. Kanellakopoulos, and P. Kokotovic, Nonlinear and Adaptive Control Design. New York: Wiley, 1995.

[17] A. Isidori, Nonlinear Control Systems. London: Springer-Verlag, 1995.

[18] H. Khalil, Nonlinear Systems. New Jersey, USA: Prentice-Hall, 1996. 